\title{
Effects of rotational speed and blade profile on mass flow at exhaust port in roots compressor
}

\author{
Yuqiang Cai ${ }^{1,2, a}$, Decai $\mathrm{Li}^{1, b}$ \\ ${ }^{1}$ Beijing Jiaotong University, Haidian, Beijing 100044, China \\ ${ }^{2}$ North China University of Science and Technology, Tangshan city, Hebei Province 063000, China \\ a781621382@qq.com , bdcli@bjtu.edu.cn
}

Keywords: roots compressor, rotor profile, rotational speed, mass flow, numerical simulation

Abstract. The roots compressor is applied widely in chemical industry as well as aerospace industry. In the article, a new combination roots compressor rotor profile curve was designed, internal flow field in three blade straight and twisted roots compressor are simulated. The results show that twisted blade roots compressor has the lower pulsation than the straight one. And the higher the speed of rotor is, the greater the compressor exhaust gas mass flow will be, but the more obvious the pulsation of the exhaust gas flow is. From the situation of the flow pulsation, the speed of the roots compressor shall not exceed 3000rpm.

\section{Introduction}

The vapor compression distillation assembly is a phase-change water recovery technology which will reclaim water from waste water. The roots compressor is a key unit which perform vapor compress. It belongs to volumetric compaction machines [1]. Although it has advantages of no inner compressing, in the meanwhile, it has disadvantages of pressure pulsation because of the high pressure vapor at the outlet flowing back to the basic-volume[2,3]. In order to reduce its pressure pulsation and improve its proficiency, a new combination profile curve is designed. A transient CFD numerical simulation is performed by means of dynamic mesh technique in ANSYS Fluent software. The effect of rotational speed and the blade profile on the compressor outlet mass flow is studied, It can provide valuable flow information to improve the roots compressor performance[4,5].

\section{The profile curve design of the roots rotor}

At present, the roots rotor mainly has three basic profile curves: circular arc profile curve, involute profile curve and cycloid profile curve. In the article, a new combination profile curve which has the advantages of area utilization factor is presented. It consist of three curves: circular arc profile curve on the tooth top, involute profile curve and the envelope profile curve on tooth root. The rotor can be either straight or twisted. Twisted roots rotor is shown in Fig. 1, and it has three twisted blade rotor.

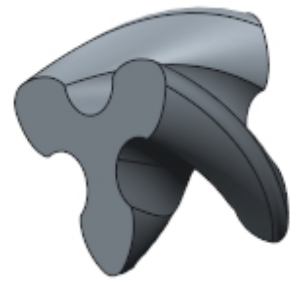

Fig. 1 twisted roots rotor

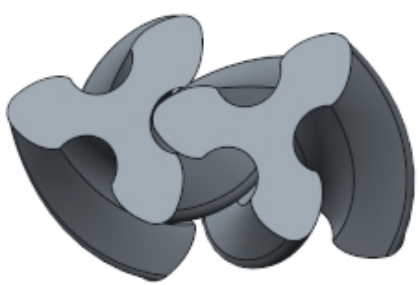

Fig.2 Assembly drawing of twisted roots rotors

The twisted roots rotor is created as follow: at first the blade profile is created in Parametric Creo 2.0 software according to the profile equation, and then a cylindrical helical curved surfaces of twisted blade is established by using cylindrical coordinates. The equation of the cylindrical helical curved surface is:

$$
\begin{array}{ll}
r=45.8 \quad & / * \text { Roots rotor pitch circle radius; } \\
\text { theta }=t * 60 \quad / * \text { twisted angle of spiral curved surface; }
\end{array}
$$


$z=t * 100 \quad / *$ The thickness of the rotor roots.

Change the torsion angle to theta $=-t * 60$, another rotor is created using the same method, the virtual assembly of the two rotor is shown in Figure 2. And it showed that there is no interference phenomenon.

\section{The finite element model of three blade twisted roots compressor}

The flow field of three blade twisted roots compressor is shown in Figure 3. The calculation model of the twisted blade roots compressor is divided into three parts: the inlet part, rotating region and outlet part. The gas inlet size, outlet size, center distance of the rotor and the gap value of the compressor are the same as the straight blade compressor. The centre distance of the roots rotor is $91.6 \mathrm{~mm}$.

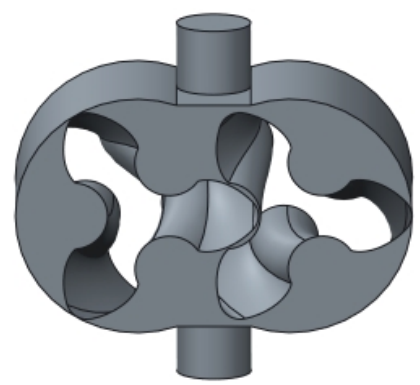

Fig.3 3D model of flow field

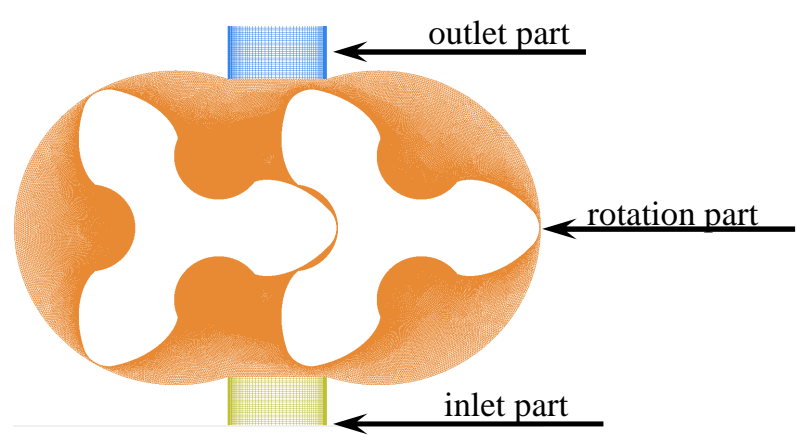

Fig. 4 The initial mesh of straight roots compressor

The initial mesh of the roots compressor of straight blade is shown in fig 4 . The initial mesh of twisted blade roots compressor is shown in fig 5. The two roots rotor wall is set to Dynamic Mesh Zone. The object of numerical simulation is three-dimensional model, the exhaust and inlet part select the hexahedral mesh, rotating region select the tetrahedral mesh. Besides, the nodes in hybrid grid will also be merged in the ANSYS ICEM CFD software, and set the boundary condition of the mixed surface as interior, to ensure that the flow information can be transfered in the form of point to point . The grid are set to $0.5 \mathrm{~mm}$ in the air inlet part and the exhaust part; the maximum size in the rotating area is $0.5 \mathrm{~mm}$, and the maximum grid size of the left and right side of the rotor is set to $0.1 \mathrm{~mm}$. Finally the initial mesh quality is reached more than 0.4 , it can meet the requirements of the calculation.

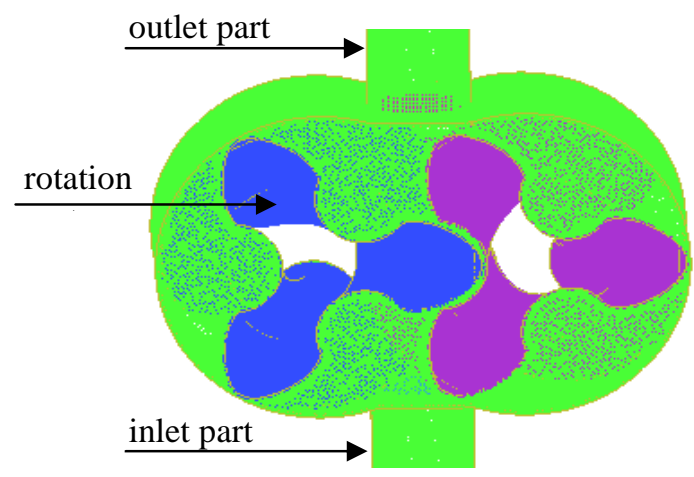

Fig.5 Initial mesh of twisted blade roots compressor

\section{The initial conditions and boundary conditions}

The rotate speed of the roots rotor is $n$, we use $n=2800 \mathrm{rpm}$, and the operating pressure is $8639 \mathrm{~Pa}$, the outlet boundary adopts pressure outlet and the inlet boundary adopts pressure inlet. The gas in the 
roots compressor is vapour. Create the $z=0$ (Iso-Surface) section and monitor the flow state of gas in the section.

\section{Numerical simulation results of the internal flow field}

Through the numerical simulation, the mass flow varying curve with the rotor angle is shown in Fig. 6 inside three blade twisted roots compressor, and the outlet mass flow curve is showed in Fig.7 inside the straight blade roots compressor. By comparison, it can be found that the flow pulsation of the twisted blade roots compressor exhaust port is lower than straight blade roots compressor. Straight blade roots compressor exhaust port flow pulsation amplitude is about $0.12 \mathrm{~kg} / \mathrm{s}$, the pulsation amplitude value of the twisted blade is about $0.04 \mathrm{~kg} / \mathrm{s}$. At moment when the base volume is connected to exhaust port of the straight blade roots compressor, the high pressure gas in the exhaust port is mixed into the original low pressure gas in the base volume because of the rapid flow of high pressure gas. But because of the spiral curved surface of the rotating twisted blade in twisted blade roots compressor, its base volume is gradually connected to the exhaust port, the return strength of high pressure gas in the exhaust port is reduced, so as to weaken the uneven of the flow pulsation of the exhaust port, there is also no negative flow.

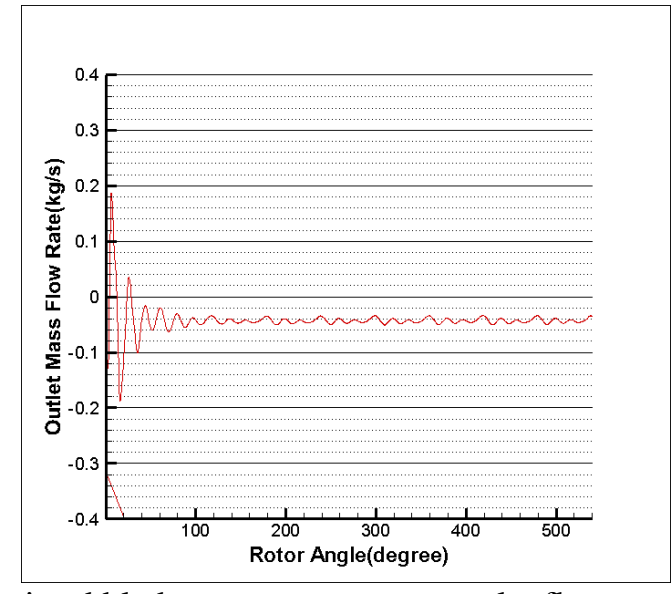

Fig.6 twisted blade roots compressor outlet flow rate

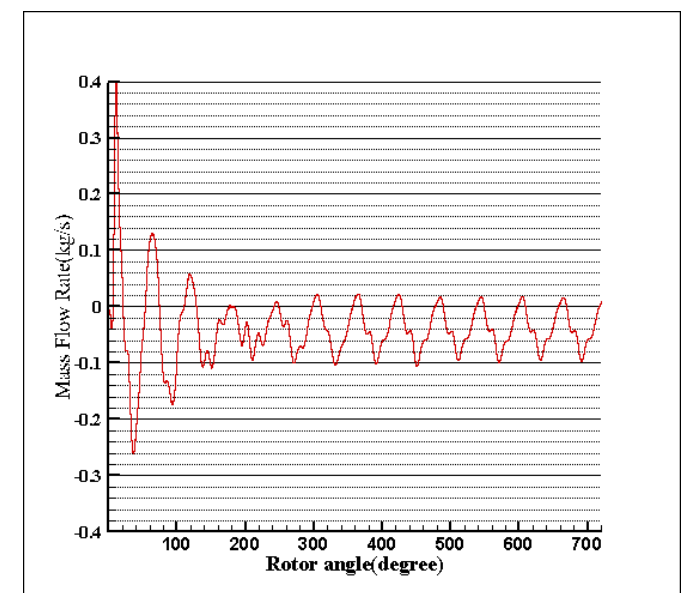

Fig.7 Straight blade roots compressor outlet flow rate

Static nephograms of the twisted blade roots compressor is shown in Fig.8. the twisted blade roots compressor will not occur the vortex impact because of the high and low pressure doped together, the static pressure inside the compressor is stable, there is no obvious pressure drop,. Thereby, the noise and vibration of the exhaust gas generated by the return shock is reduced to a large extent. Therefore, the noise reduction performance of the twisted blade roots compress is more superior than the straight blade roots compressor.

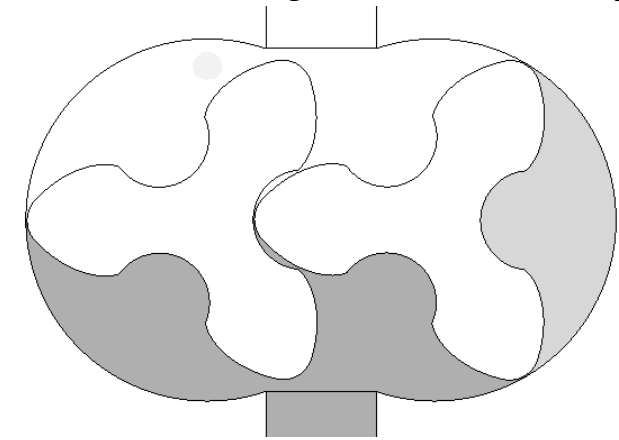

(a) $\theta=60^{\circ}$

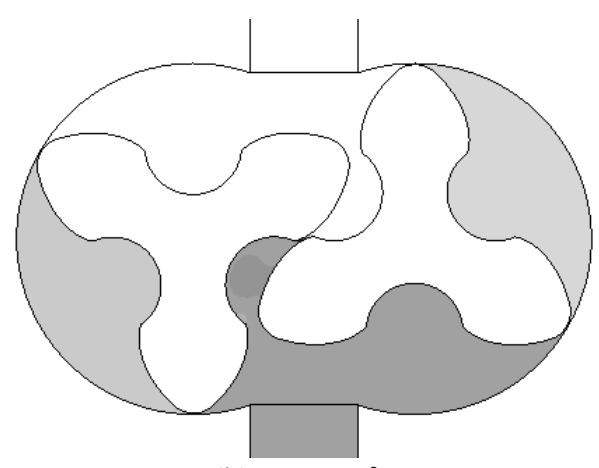

(b) $\theta=90^{\circ}$

Fig.8 Static nephograms of the twisted blade roots compressor horizontal center surface 


\section{The effect of speed on the roots compressor outlet mass flow}

In order to observe the effects of different speed of roots compressor on compressor outlet flow, compressor noise and vibration characteristics, in this section, numerical calculation is carried out respectively when the speed of roots compressor is $1500 \mathrm{rpm}, 2000 \mathrm{rpm}, 2500 \mathrm{rpm}, 3000 \mathrm{rpm}$ and $3500 \mathrm{rpm}$, the curves of roots compressor exhaust port mass flow with the rotor angle under different speed is shown in Fig.9.

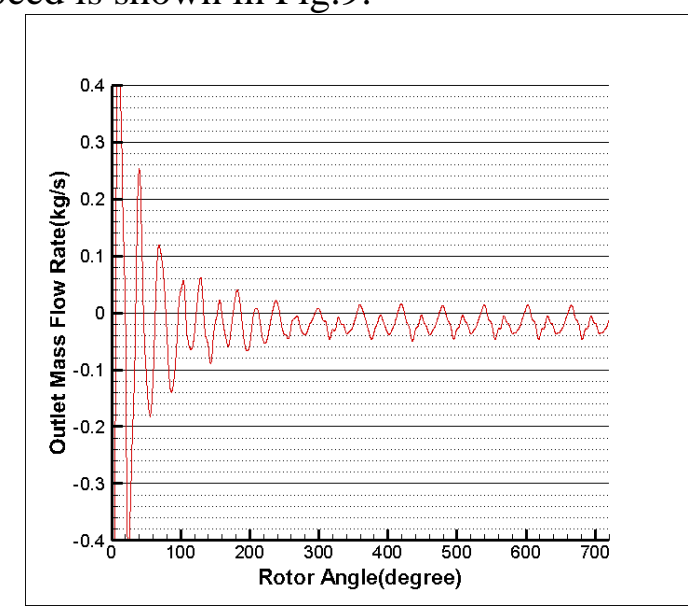

(a) $n=1500 \mathrm{rpm}$

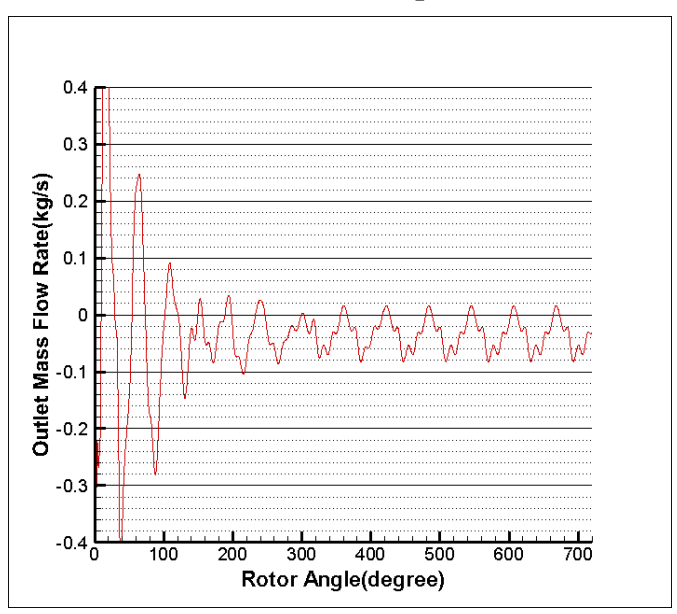

(c) $n=2500 \mathrm{rpm}$

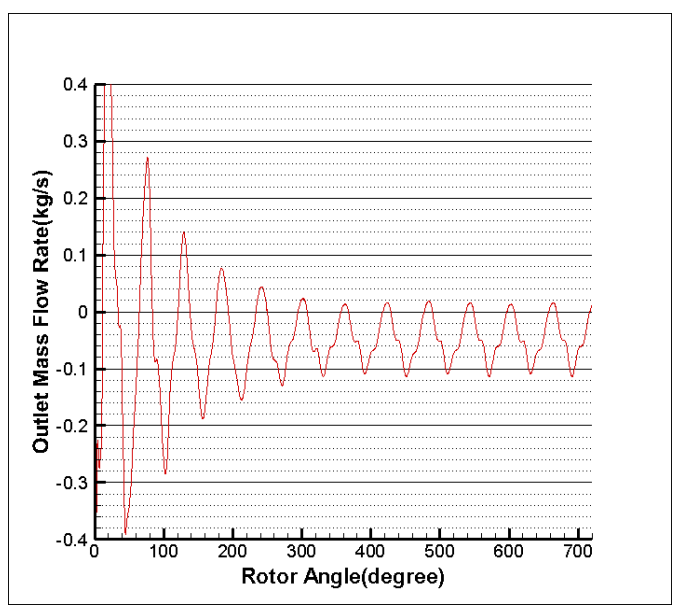

(e) $n=3000 \mathrm{rpm}$

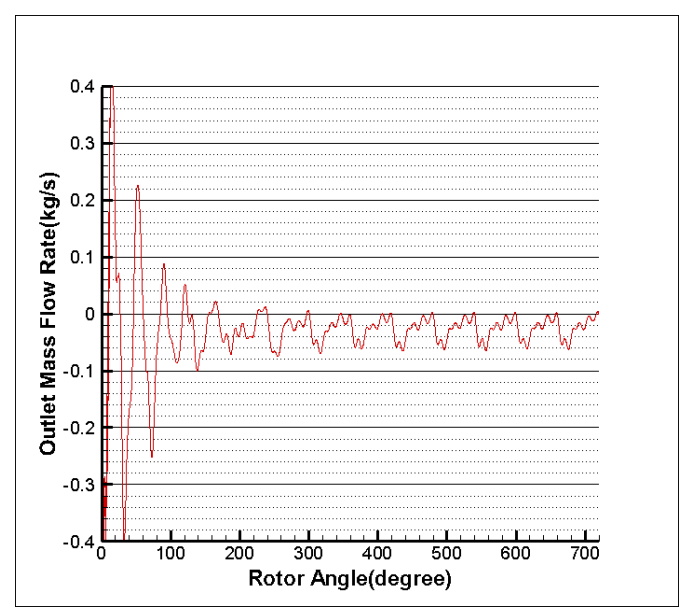

(b) $n=2000 \mathrm{rpm}$

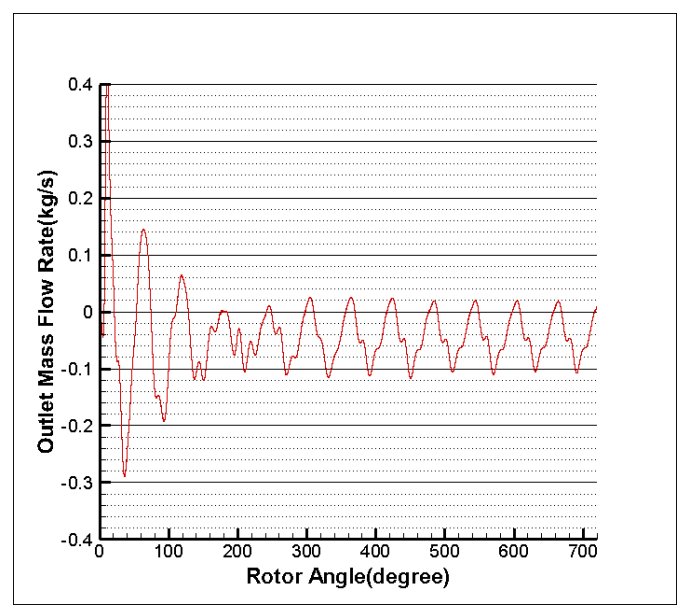

(d) $n=2800 \mathrm{rpm}$

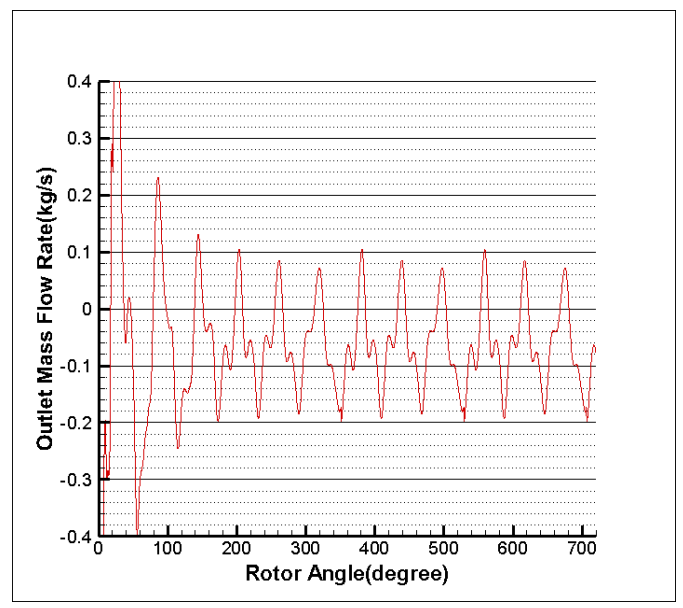

(f) $n=3500 \mathrm{rpm}$

Fig.10 Diagrams of outlet mass flow rate at different speeds

It can be seen from the figure that the speed level does not affect the periodic variation of the exhaust port flow, the rotational speed and exhaust flow of roots compressor is positively related, the higher the speed of the compressor is, the greater the average flow of the exhaust port will be. The 
speed of the compressor is the main factor to determine the compressor exhaust gas mass flow.

However, the flow pulsation range of the compressor outlet is greater when the speed is higher, which will cause the vibration and aerodynamic noise. Therefore, from the situation of the flow pulsation, the speed of the roots compressor shall not exceed 3000rpm.

\section{Conclusion}

In this chapter, firstly the three-dimensional model and the finite element model of three twisted blade roots compressor as well as straight blade compressor are created, and the internal flow field of the two kind compressor is simulated. By comparison, it can be obtained that that the exhaust mass flow of the three twisted blade roots compressor is more stable, fluctuating range is small, and its noise reduction performance is better.

Secondly, the three straight blade roots compressor internal flow field are simulated under different speed, It can be found that the higher the speed is, the the greater the compressor exhaust gas mass flow will be, the more obvious the pulsation of the exhaust gas flow is.

\section{Acknowledgements}

This work was financially supported by the Hebei Province Advanced Personnel Training Foundation (A201400214).

\section{References}

[1] Dingguo Zhang, Pengcheng Shu. Rotary Compressor. Beijing: Mechanical industry press, 1982. In Chinese.

[2] R.W. Fox, P.J. Pritchard, A.T. McDonald. Introduction to Fluid Mechanics. (seventh ed.)Wiley, New York (2009)

[3] Fujun Wang, Beijing: Computational fluid dynamics analysis-- Principle and application of CFD software Tsinghua University press, 2004.09. In Chinese.

[4] Hougen Liu, Chengjia Li, Yongsheng Liu. Improvement Study on Involute Profile of Roots Mechanical Supercharger Rotors[J], Fluid machinery, 2013,08:48-52+39. In Chinese.

[5] P.WIELAND, C.HUTCHENS, D.LONG. Final report on life testing of the vapor compression distillation/urine processing assembly (VCD/UPA) at the Marshall Space Flight Center (1993-1997), NASA/TM-1998-208539. 